\title{
Universality in Relativistic Aspirations of Helmholtz Soliton Pulses
}

\author{
Gemma Lancaster, Mark J. Lundie, Timothy F. Hodgkinson, James M. Christian, Graham S. McDonald \\ Materials \& Physics Research Centre, University of Salford, Greater Manchester, M5 4WT, U.K.
}

Since the heady days of pioneering temporal optical soliton research [1-3], pulse propagation equations have been almost exclusively of the nonlinear Schrödinger form. These parabolic models are derived from Maxwell's equations through a careful handling of the linear dispersion operator, and invoking the near-universal slowlyvarying envelope approximation (SVEA). A more general approach to pulse evolution, and hence a potentially more accurate one, may clearly be adopted by seeing what progress can be made when the SVEA is relaxed. When one does so, the governing equation is of the Helmholtz (elliptic or hyperbolic) type. While the precedent of using these more sophisticated models was set in the late 1970s [4], they appear to have received relatively little subsequent attention in the literature. A notable exception is the recent paper by Biancalana and Creatore [5], giving Helmholtz-type pulse models a new physical context in the guise of spatial dispersion.

Here, we take the first steps toward redressing this imbalance by presenting a compact description of Helmholtz optical pulse evolution in waveguides. An overview of our most recent research into universal nonlinearities will be given; classic models of greatest relevance in waveguide optics include Kerr [1-3,6], cubic-quintic [7] and saturable [8]. Physical and mathematical self-consistency demand one pays careful attention to the intrinsic frame-of-reference character of the propagation problem. Exact analytical solitons will be reported, derived using known Helmholtz spatial solutions $[9,10]$ as a starting point. Extensive computations also confirm the status of the new temporal solitons as robusts attractors (see Fig. 1).
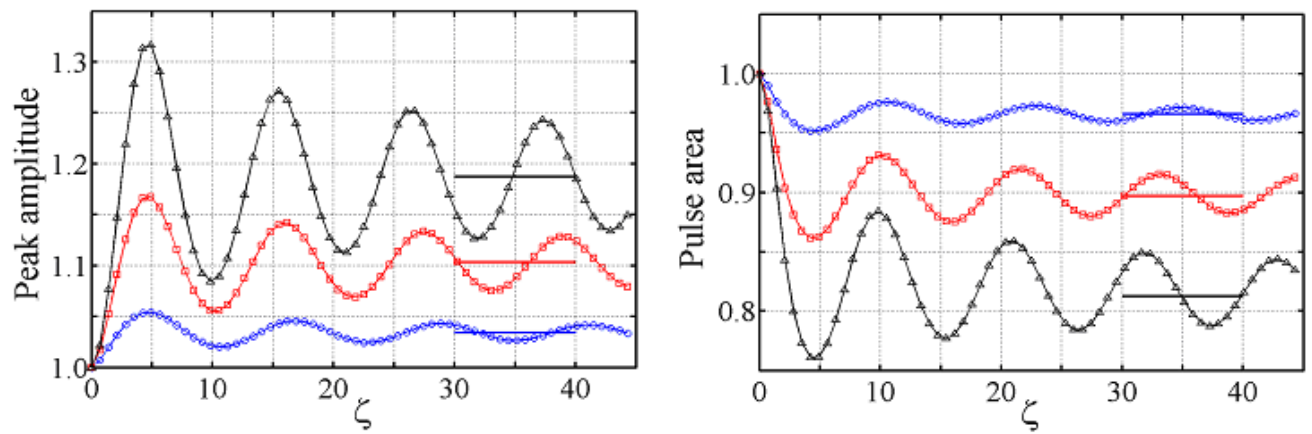

Fig. 1 Self-reshaping oscillations of perturbed soliton pulses (horizontal bars denote asymptotic theoretical predictions).

One of the most intriguing results to emerge from our analyses is a formal correspondence between Helmholtz-type governing equations and Einstein's special theory of relativity. This connection is manifest through geometric (space-time) transformation laws that are reminiscent of the familiar Lorentz transformation. It also appears in the velocity combination rule, which bears striking resemblance to that in relativistic kinematics. These key properties are tightly connected to the structure of the linear wave operator, being independent of both the system nonlinearity and the particular solutions under consideration (e.g., continuous waves or solitons). Interestingly, conventional pulse theory $[1-4,7,8]$ is recovered from the Helmholtz formalism in a manner akin to the 'low speed' emergence of Newtonian mechanics from special relativity.

\section{References}

[1] V. E. Zakharov and A. B. Shabat, "Exact theory of two-dimensional self-focusing and one-dimensional self-modulation of waves in nonlinear media," Sov. Phys. JETP 34, 62 (1972).

[2] A. Hasegawa and F. Tappert, "Transmission of stationary nonlinear optical pulses in dispersive dielectric fibres. I. Anomalous dispersion," Appl. Phys. Lett. 23, 142 (1973).

[3] L. F. Mollenauer, R. H. Stolen, and J. P. Gordon, "Experimental observation of picosecond pulse narrowing and solitons in optical fibres," Phys. Rev. Lett. 45, 1095 (1980).

[4] Kh. I. Pushkarov, D. I. Pushkarov, and I. V. Tomov, "Self-action of light beams in nonlinear media: soliton solutions," Opt. Quantum Electron. 11, 471 (1979).

[5] F. Biancalana and C. Creatore, "Instabilities and solitons in systems with spatiotemporal dispersion," Opt. Express 16, 14882 (2008).

[6] J. M. Christian, G. S. McDonald, T. F. Hodgkinson, and P. Chamorro-Posada, "Bright solitons of a cubic Helmholtz pulse equation," submitted 2011

[7] J. Hermann, "Bistable bright solitons in dispersive media with a linear and quadratic intensity-depending refraction index change," Opt. Commun. 87, 161 (1992).

[8] W. Krolikowski and B. Luther-Davies, "Analytic solution for soliton propagation in a nonlinear saturable medium," Opt.Lett. 17, 1414 (1992).

[9] P. Chamorro-Posada, G. S. McDonald, and G. H. C. New, "Non-paraxial solitons,” J. Mod. Opt. 45, 1111 (1998).

[10] J. M. Christian, G. S. McDonald, and P. Chamorro-Posada, "Bistable Helmholtz bright solitons in saturable materials," J. Opt. Soc. Am. B 26, 2323 (2009). 